\title{
Co-infection of enterovirus 71 and Staphylococcus aureus in a 6-month-old male infant
}

Peng Hu, Jian Wang, Hai Bo Kong, Xue Qi Zhao, Jie Cai, Bo Hu

Department of Pediatrics, the First Affiliated Hospital of Anhui Medical University, Hefei, China

Submitted: 22 January 2014

Accepted: 3 February 2014

Arch Med Sci 2015; 11, 4: 905-907

DOI: 10.5114/aoms.2015.53314

Copyright $\odot 2015$ Termedia \& Banach

Enterovirus 71 (EV71), as a member of the Picornaviridae family, is characterized by a single positive-strand genomic RNA known to cause hand, foot, and mouth disease (HFMD) in children. Since the first identification of EV71 infection in California in 1969, periodic epidemics have broken out worldwide [1]. In China, the first EV71 strain was isolated in 1987 by Zheng et al. [2]. Generally speaking, EV71 infection follows a benign and self-limiting course with multiple vesicles on the hands, feet and buccal mucosa. However, over the last decade EV71-associated HFMD has posed a great economic and social burden because of more frequent outbreaks with serious complications including neurological involvement, myocarditis and pulmonary edema [3, 4]. Liu et al. carried out an epidemiological analysis of an HFMD outbreak in 2010 in Nanchang city, and found that $12(11 \%)$ patients were co-infected with both EV71 and coxsackievirus A16 (CA16) [5]. In the present report, we describe an unusual case of co-infection of EV71 and Staphylococcus aureus (SA).

A 6-month-old male infant with a 4-day history of general malaise, fever, poor feeding and skin lesions was admitted to the Department of Pediatrics at the First Affiliated Hospital of Anhui Medical University in June 2012. The birth and past medical history were non-significant, and the family had no history of similar disorders. On admission, his blood pressure was 70/35 $\mathrm{mm} \mathrm{Hg}$, heart rate was 132 beats per minute, axillary temperature was $39.2^{\circ} \mathrm{C}$, and $\mathrm{O}_{2}$ saturation (pulse oximetry) was $95 \%$. Physical examination revealed the presence of sporadic pustules and papules on his lower limbs, buccal and perianal mucosa (Figure 1). There was no lymphadenopathy. Cardiac and pulmonary physical examinations were unremarkable. The abdomen showed no clinical signs of peritoneal irritation, masses, or enlarged organs. EV71 was identified from both throat swab and pustular fluid by virus isolation techniques. In addition, two blood cultures from samples obtained at admission were both positive for SA. His initial blood counts were as follows: white blood cell $19.62 \times 10^{9} /$ with $63.7 \%$ neutrophils, $21.3 \%$ lymphocytes, and $8.1 \%$ monocytes, hemoglobin $127 \mathrm{~g} / \mathrm{l}$, and platelet $361 \times 10^{\%} / \mathrm{l}$. C-reactive protein was $27 \mathrm{mg} / \mathrm{l}$. Serum biochemistry and urinary analysis were normal. Echocardiogram demonstrated no pathologic findings. The patient was diagnosed with co-infection of EV71 and SA, mainly according to the results of the physical examination, virus isolation and blood cultures. He was treated with intravenous acyclovir, immunoglobulin and vancomycin, and bed rest for 14 days. The fever and skin lesions gradually resolved.

\author{
Corresponding author: \\ Peng Hu \\ Department of Pediatrics \\ the First Affiliated Hospital \\ of Anhui Medical University \\ No. $218 \mathrm{Ji}$-Xi Road \\ Hefei 230022, PR China \\ Phone: +8655162922058 \\ Fax: +8655163633742 \\ E-mail: hupeng28@aliyun. \\ com
}



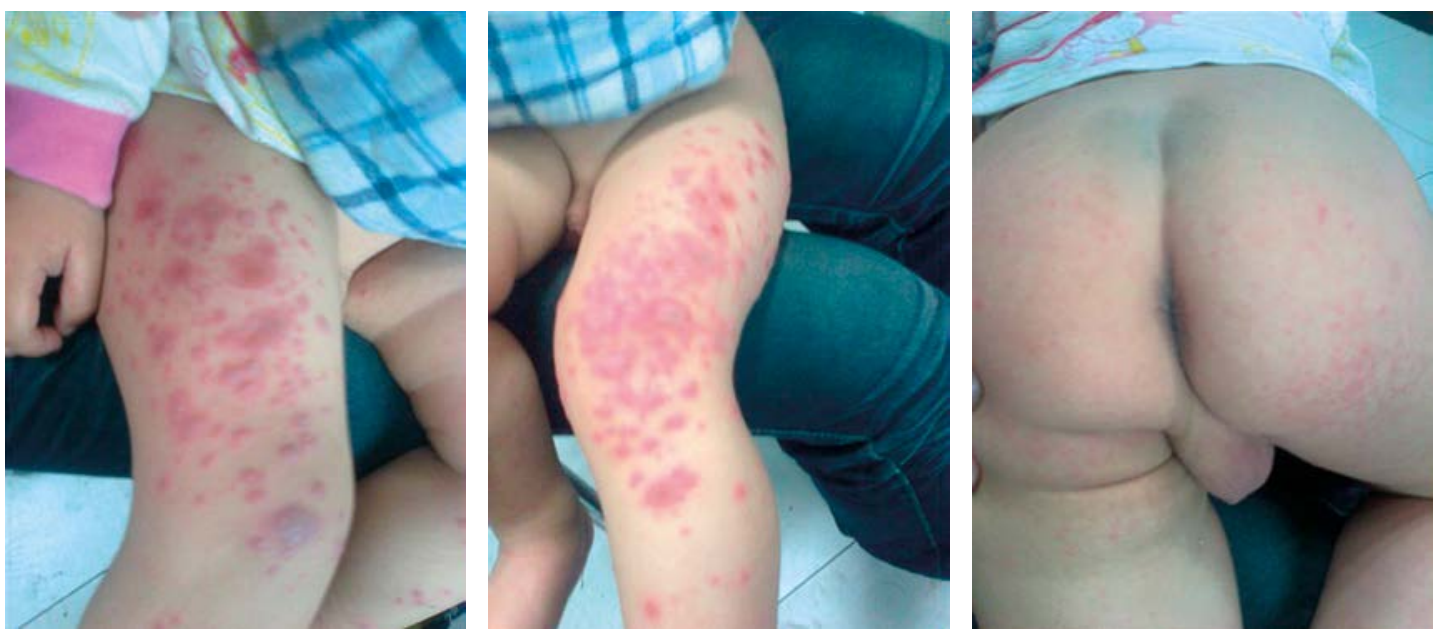

Figure 1. Sporadic pustules and papules on lower limbs and perianal mucosa

The latest large epidemic of EV71-associated HFMD occurred in China in 2008. At the epicenter in Fuyang city, Anhui province, of the 6,049 cases reported between March 1 and May 9, 2008, 3,023 patients were hospitalized, 353 cases were severe and 22 were fatal [6]. The diagnosis of EV71 infection relies mainly on laboratory identification, because not all patients have characteristic skin lesions [7]. The combination of throat swab plus vesicle fluid is most useful, and can increase isolation rates from $49 \%$ with throat swab alone, and $48 \%$ with vesicle fluid alone, to $67 \%$ [8]. EV71 is related to more serious complications. The risk factors contributing to severe HFMD are young male children ( $\leq 2$ years old), general malaise, peak temperature $\geq 38.5^{\circ} \mathrm{C}$, atypical physical findings (tachycardia, tachypnea, hypertension, pulmonary hemorrhage and limb movement disorder), leukocytosis and hyperglycemia [9, 10]. Therefore, our case was classified as severe due to the existence of several risk factors, including the fact that the patient was a 6-month-old male, with general malaise, peak temperature $\geq 38.5^{\circ} \mathrm{C}$ and leukocytosis.

In the present report, we describe for the first time an unusual case of co-infection of EV71 and SA. Staphylococcus aureus, a Gram-positive bacterium, is one of the most common causes of both healthcare- and community-acquired infections. A large, multi-center study demonstrated that incidence of SA bacteremia in the United States ranged between 19 and 40 cases per 100,000 with the case fatality rates ranging from $19 \%$ to $24 \%$ [11]. However, the prevalence of SA infection in China is still unknown. A broad variety of infections, ranging from minor infections of the skin to severe infections such as bloodstream infections and endocarditis, can be caused by SA [12]. In the present case, the chief clinical demonstrations were general malaise, fever, pustules, leukocytosis, elevation of C-reactive protein, and a positive blood culture, which might be attributed, in part, to skin and bloodstream infections. In addition, both cardiac physical examination and echocardiogram were normal in our patient; thus there was no evidence for the existence of endocarditis. Decreased host immunity in this EV71-infected infant may place him at increased risk of SA infection. More persuasively, Furuno et al. identified 131 individual episodes of SA bacteremia among HIV-positive patients during the study period between January 1 , 2003 and December 31, 2005 [13]. Furthermore, we speculate that individual host genetic factors may also contribute to co-infection of EV71 and SA.

Although the pathogenesis of EV71 infection is still not fully understood, and therapeutic effects of antiviral drugs are controversial, the clinical outcome of our patient was satisfactory after intravenous acyclovir, immunoglobulin and vancomycin, and bed rest for 14 days. Vancomycin is now recommended empirically for SA infection [14]. Besides this, several clinical investigations have also indicated that the use of intravenous immunoglobulin can significantly reduce the acute mortality in patients with either EV71 or SA infection $[15,16]$.

\section{Conflict of interest}

The authors declare no conflict of interest.

\section{References}

1. Schmidt NJ, Lennette EH, Ho HH. An apparently new enterovirus isolated from patients with disease of the central nervous system. J Infect Dis 1974; 129: 304-9.

2. Zheng ZM, Zhang JH, Zhu WP, He PJ. Isolation of enterovirus type 71 from the vesicle fluid of an adult patient with hand-foot-mouth disease in China. Virologica Sinica 1989; 4: 375-81.

3. Ng DK, Law AK, Cherk SW, Mak KL. First fatal case of enterovirus 71 infection in Hong Kong. Hong Kong Med J 2001; 7: 193-6.

4. Hu P, Hou S, Du PF, Li JB, Ye Y. Paroxysmal supraventricular tachycardia in an infant with hand, foot, and mouth disease. Ann Dermatol 2012; 24: 200-2. 
5. Liu MY, Liu W, Luo J, et al. Characterization of an outbreak of hand, foot, and mouth disease in Nanchang, China in 2010. PLoS One 2011; 6: e25287.

6. Zhang Y, Zhu Z, Yang W, et al. An emerging recombinant human enterovirus 71 responsible for the 2008 outbreak of hand foot and mouth disease in Fuyang city of China. Virol I 2010; 7: 94.

7. Xu W, Liu CF, Yan L, et al. Distribution of enteroviruses in hospitalized children with hand, foot and mouth disease and relationship between pathogens and nervous system complications. Virol J 2012; 9: 8.

8. Ooi MH, Solomon T, Podin Y, et al. Evaluation of different clinical sample types in diagnosis of human enterovirus 71-associated hand-foot-and-mouth disease. J Clin Microbiol 2007; 45: 1858-66.

9. Ooi MH, Wong SC, Mohan A, et al. Identification and validation of clinical predictors for the risk of neurological involvement in children with hand, foot, and mouth disease in Sarawak. BMC Infect Dis 2009; 9: 3.

10. Li Y, Zhu R, Qian Y, Deng J. The characteristics of blood glucose and WBC counts in peripheral blood of cases of hand foot and mouth disease in China: a systematic review. PLoS One 2012; 7: e29003.

11. Fridkin SK, Hageman JC, Morrison M, et al. Methicillin-resistant Staphylococcus aureus disease in three communities. N Engl J Med 2005; 352: 1436-44.

12. Naves KS, Trindade NV, Gontijo Filho PP. Methicillin-resistant Staphylococcus aureus bloodstream infection: risk factors and clinical outcome in non-intensive-care units. Rev Soc Bras Med Trop 2012; 45: 189-93.

13. Furuno JP, Johnson JK, Schweizer ML, et al. Community-associated methicillin-resistant Staphylococcus aureus bacteremia and endocarditis among HIV patients: a cohort study. BMC Infect Dis 2011; 11: 298.

14. Rahikka P, Syrjänen J, Vuento R, Laine J, Huttunen R. Meticillin-resistant Staphylococcus aureus (MRSA) bacteraemia in Tampere University Hospital: a case-control study, Finland October 2002 to January 2010. Euro Surveill 2011; 16: 19958.

15. He YX, Fu D, Cao DZ, Liu HY, Huang QL, Li CR. Critical care and therapy based different illness state of $80 \mathrm{pa}-$ tients with severe hand-foot-and-mouth disease seen in Shenzhen. Zhonghua Er Ke Za Zhi 2009; 47: 338-43.

16. Rajam G, Hammons GM, Carlone GM, Sampson JS, Ades EW. A novel innate immune-enhancement strategy combined with IVIG rescues mice from fatal Staphylococcus aureus septicemia. Int I Microbiol 2011; 2011: 725483. 